\title{
Soft features for robotics
}

\author{
Innovations in soft materials design and engineering are delivering promising functional components for advanced
} soft robotic applications.

( ver the past decade, research developments have provided a myriad of artificial soft materials with lifelike functionalities, including sensing and response mechanisms $s^{1,2}$, locomotion $^{3}$ and even autonomy ${ }^{4}$. These functional soft materials have made a tremendous impact in the field of soft robotics, which aims to deliver safe, reliable and versatile robotic systems for close collaboration with humans to tackle broad challenges facing humankind, from healthcare to sustainability. In this Focus issue, we gather several research studies and a Comment article to highlight the contribution of innovation in functional soft materials to shaping the future of soft robots.

Bioinspired soft robotic systems that mimic or outperform living functions and capabilities have been developed to solve problems that are difficult or even impossible to be addressed by traditional 'hard' robots ${ }^{5}$. Autonomous behaviour is a fundamental feature of living organisms, whose translation into artificial systems has proved to be challenging. In an Article in this issue of Nature Materials, Alfred Crosby and colleagues describe design principles for producing self-repeating movements in polymeric materials. Inspired by the instant prey-catching grasp of carnivorous plants, they harnessed the mechanical instabilities of a slowly drying polymer to generate discrete but continuous snap-through buckling events. These elastic deformation iterations release enough energy to make polymer strips wiggle and polymer discs jump high enough to climb steps. As discussed in an accompanying News \& Views article by Johannes Overvelde, while these self-repeating jumping discs cannot be regarded as fully autonomous soft robots (due to the lack of sensing and feedback mechanisms), the work highlights the potential of hysteresis in the response to create useful functionalities in soft robots, especially when integrated in engineered designs to allow for sensing and control over the triggering of such response. In another example of sustained motion by soft materials, Dirk Broer and Ghislaine Vantomme demonstrate in their Article how two connected polymeric actuators display collective synchronization when stimulated by light. Rectangular

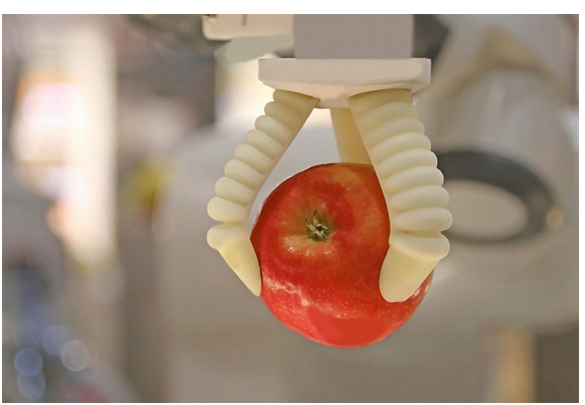

Credit: FC_Italy / Alamy Stock Photo

liquid-crystalline-network films containing an azobenzene chromophore display light-fuelled oscillations with a frequency set by their size and mechanical properties. When two actuators of this type are connected by a strip of the same material, their movements become entangled leading to synchronized in-phase and anti-phase oscillations. This coupling, evocative of the historical observations by Christiaan Huygens on the synchronization of pendulum clocks $^{6}$, may be useful for the development of soft robotic systems that replicate the synchronization and collective motion of their biological counterparts, as highlighted in the accompanying News \& Views article by Yanlei Yu.

The establishment of safe and straightforward interactions between robotic systems and humans is one of the main challenges in robotics. Advances in the complexity and versatility of functional soft materials are changing the way that researchers are addressing this issue ${ }^{7}$. In an Article also in this issue, Lilian Hsiao and colleagues present a physical description of the friction between soft, lubricated and patterned surfaces sensed by a human finger with its natural oil and sweat layers rubbing on the solid surface. Such elastohydrodynamic lubrication friction results from an intricate mechanism involving solid deformation, fluid dissipation and localized flows that is highly dependent on surface pattern geometry. This knowledge about surface texture properties and friction can be useful for the design of improved haptic systems and soft robotic graspers that are fully functional even when the objects that they are interacting with are wet and dirty.
Soft materials with improved mechanical properties and advanced functionalities have been fundamental for the development and establishment of soft robotics as a field of research. In a Comment article, Christoph Keplinger and colleagues discuss how the future of soft robotics will need further innovations in functional soft materials to deliver safe, reliable and highly capable robots that can truly revolutionize our daily lives. A major bottleneck in achieving such technologies lies in the fact that functional soft materials often still perform well below their full potential, as predicted by their underlying working principles. Such underperformance is further noted when these materials are incorporated into multicomponent robotic systems, since each module has completely distinct mechanical properties and responses to the same stimuli. These performance and interface issues will require substantial efforts and innovation from the materials science community to attain functional soft materials that perform at their full potential both in isolation and when integrated into fully functional robots. These researchers thus encourage material scientists with interest in the soft robotics field to tackle these challenges and provide innovative functional soft materials for robotic systems that exceed the capabilities of currently available solutions.

The path for achieving compliant, adaptable robots with super-human capabilities such as those portrayed in science fiction is still long. Despite considerable progress in terms of design, intelligence and functionalities, it is clear that many of the hurdles that lie ahead are at their core a material problem. Further research and collaboration between academia and industry is required to fully integrate soft robotics into our daily lives.

Published online: 23 November 2021 https://doi.org/10.1038/s41563-021-01165-2

References

1. Liu, C. et al. Science 372, 1078-1081 (2021).

2. Wang, Z. et al. Nat. Mater. 20, 869-874 (2021).

3. Hu, W. et al. Nature 554, 81-85 (2018).

4. Wehner, M. et al. Nature 536, 451-455 (2016)

5. Majidi, C. Soft Robot. 1, 5-11 (2014).

6. Bennett, M. et al. Proc. R. Soc. A 458, 563-579 (2002).

7. Yuk, H., Lu, B. \& Zhao, X. Chem. Soc. Rev. 48, 1642-1667 (2019). 